\title{
Measurement Method of Automobile Body Inclination Angle Based on Headlamp Area Detection
}

\author{
Peijiang Chen ${ }^{*}$
}

\author{
School of Automobile, Linyi University, Shandong, 276000, China
}

\begin{abstract}
In the standards about the contour dimension of automobile in most countries, the height difference of body symmetrical points or body inclination angle of automobile was specified. To improve the vehicle performance and operation safety, a measurement method of car body inclination angle was studied. According to the camera calibration and the distance between camera and automobile, the front license plate detection area was estimated, and the accurate license plate positioning was realized based on the constraint on length to width ratio. According to the statistics of distance between license plate and headlamps, the headlamp detection areas were estimated. The feature points of the headlamps were extracted based on the angle and distance constraints of lines, and then the height difference of the symmetrical headlamp feature points were analyzed and the automobile body inclination angle was gotten. The experiment results showed that this method effectively detected the automobile inclination angle, and the measurement could provide data support to improve car's usage performance and lay foundation for the next automobile detecting applications. It was a non-contact measurement method that could realize automatic detection and easily combined with other testing projects.
\end{abstract}

Keywords: Automobile body, Headlamp area detection, Hough transform, Inclination angle.

\section{INTRODUCTION}

As one of the most important transport means, automobile has a history more than 100 years. Because of flexibility, convenient manipulation, it is closely related and bound together inseparably with people's lives, changing people's lifestyles in many ways and expanding the range of activities to a large extent. Meanwhile, the design and manufacture of automobile are important basic industries and occupy important position in the country's economy.

After long time use, the automobile application performances, including power and reliability, deteriorate and the failure rate increases. With the progress of automobile technologies, more countries continuously improve the relevant technical regulations and put forward new requirements for the technologies of automobile detection and fault diagnosis. As a result, the number of detection items increases, the testing content tends to be delicate, the testing standard becomes strict, and the detection of some detail parts of automobile body also increasingly arouses people's attention.

For automobile appearance, the inclinatiion of automobile body has attractd more attention. Body inclination means that the height difference between the left and right skirts of an automobile body and the ground exceeds the standard, that is, the height difference between the left and right symmetrical points of an automobile body is too large. The body inclination can be caused by many reasons: the materials of left and right leaf springs are different; the bot- tom beam positioning has deviation when making body frame; body frame has deformation; when body and chassis are fitted together with each other, their centerlines are not in the same vertical plane; left and right mass distribution are unbalanced when designing body layout. In addition, some behaviors violating the operation specification, such as severe overloading, long term lack of necessary maintenance, etc., can also directly or indirectly lead to automobile body inclination [1]. Of course, the uneven parking space can lead to body inclination visually.

When an automobile body inclines significantly, it affects not only the apparence, but also the car's braking performance and handling stability [2]. Furthermore, the load distribution on left and right tires is uneven, the adhesions of the tires are not the same, and thus the automobile is easy to pull to one side when braking. The inclination angle is also an important parameter relating to handling stability and ride performance of the automobile. The excessive inclination angle can make the drivers and passengers feel instable and insecure. What's more, the center of gravity of the vehicle shifts to one side, the action center of air resistance also offsets, then the lateral stability becomes worse and the automobile is easy to roll over when steering, causing accidents and losses of personal and property.

The basis of measuring the automobile inclination angle is to analyze the height difference between the symmetrical points of the automobile body, which measurement methods can be divided into two categories, contact measurement and non-contact measurement [3]. The conventional height difference measurement method is to use tape, or surveyor's pole, and other auxiliary tools. Such methods are simple, but the precision and accuracy are low, and the measuring process is inconvenient and time-consuming when the automo- 
bile body has arc shape. In order to improve the accuracy and automation degree of the measurement, with the help of the advanced information technologies and artificial intelligence technologies [4-8], researchers have carried out relevant works and developed some new techniques and methods.

Lou and Ma [9, 10], Zhao [11], et al., designed a height difference tester for automobile body, which mechanical system consisted of three components: the part of column and base, box-shaped sliding component and laser. The basic principle is to use laser reflection theory to measure the height of left and right feature points of the automobile body, this method can improve the measurement accuracy and efficiency. However, it requires that the laser must have sufficient freedom degree, can rotate in the entire circumference with $360^{\circ}$, can move freely up and down, and the laser must be in absolute horizontal status, otherwise the measurement result has relatively large errors.

In order to change the current situation that the conventional detection equipment could not quickly and accurately measure the height difference of automobile body symmetrical points, Zhang and Su [12] designed a non-contact automobile body symmetrical point height difference tester based on Solidworks software platform. The mechanical system is composed of camera's horizontal slewing device and other components. The tester uses the height difference of two measurements in the vertical direction to realize the detection task quickly and accurately. This method has high reliability and accuracy, but it has disadvantages that the operating process is complex and it cannot be fully automated.

According to the features of the height difference detection technology of automobile body symmetrical points, starting from image feature extraction and matching, camera calibration and other key technologies, Lin [13] designed a detection system based on stereo vision to realize the automatic measurement of height difference. When selecting the symmetrical points in the automobile body, the two cases, trucks and cars, were studied. The vehicle type should be pre-determined which limits its application.

Through the above analysis, these detection methods have their virtues and faults, and cannot solve the problem completely. According to the actual need of vehicle detection, an automobile body inclination angle measuring system based on digital image processing technology is designed in this study. In order to reduce the system cost, single camera is used. The system makes the computer as the center, the camera is set in front of the tested automobile, and these two devices are aligned by camera calibration. The automobile front-face image is captured by the camera. The license plate of automobile is detected and positioned based on Hough transform constrained by the ratio of height to width. The headlamp detection areas are prepositioned based on the detected license plate. According to the characteristics of headlamp shapes, the headlamp symmetrical points are extracted based on Hough transform constrained by angle and distance. The height difference of automobile body symmetrical points is analyzed and the body inclination angle is gotten. This study can lay foundation for the next automobile detection and application, and provide data support to improve the usability of automobile.
Table 1. The body widths of five kinds of representative family cars.

\begin{tabular}{|c|c|c|c|}
\hline No & Card Brand & Car Type & Width (Unit: mm) \\
\hline \hline 1 & VW & Santana & 1706 \\
\hline 2 & Toyota & Corolla & 1760 \\
\hline 3 & Chery & A3 & 1794 \\
\hline 4 & Ford & Focus & 1840 \\
\hline 5 & Audi & A6 & 1874 \\
\hline
\end{tabular}

\section{DESIGN OF AUTOMOBILE BODY INCLINATION ANGLE DETECTION SCHEME}

\subsection{Requirement of Automobile Body Inclination Angle Detection}

According to relevant standards about vehicle testing in China, the whole equipment of the vehicle should be complete, perfect and effective and all the connected parts should be tight and intact; the automobile body should be upright and the height difference of left and right symmetrical parts in outer edge of the car body should not exceed $40 \mathrm{~mm}$ when measuring from the points which are within $1.5 \mathrm{~m}$ above the ground [14]. But the standard does not specify the requirement for the body inclination angle. Then, in order to analyze the body inclination angle, the automobile body width should be known.

The vehicle width is the distance between the two planes of left and right projecting parts which are parallel to the longitudinal symmetrical plane. Put simply, it is the distance between the two extreme points across the width. Among them, the protruding parts on both sides do not include rearview mirrors, lateral signal lights, folding pedals, deformed part contacted with ground, and so on. The body width principally affects the passenger space and steering flexibility. A wide body can let passengers feel comfortable, but it reduces the convenience of driving and parking. At the same time, the car width cannot be too narrow, or it makes the passengers feel crowded, especially long time travel lets people tired. Chinese national standard requires that the width of ordinary passenger vehicle should be not greater than $2.5 \mathrm{~m}$ [15]. In fact, few cars have $2.5 \mathrm{~m}$ width. In general, for ordinary cars, if the three seats in transversal arrangement need broad riding sense, the general vehicle width is $1.8 \mathrm{~m}$ or so. The body widths of five famous brands of family cars are listed, as shown in Table $\mathbf{1}$.

As can be seen from Table 1, the body width of the five types of ordinary family cars are about $1.8 \mathrm{~m}$ and the mean is $1794.8 \mathrm{~mm}$. By comprehensive consideration, the standard body width of the ordinary car is set to $1.8 \mathrm{~m}$, which corresponding inclination angle of the automobile body can be gotten as follows.

$\alpha=\arctan (40 / 1800)=1.273^{\circ}$

That is, if the body inclination angle is smaller than $\alpha$, then it is qualified, otherwise, the reasons of car inclination should be looked for and maintained to improve the operational performance and enhance the driving safety. 


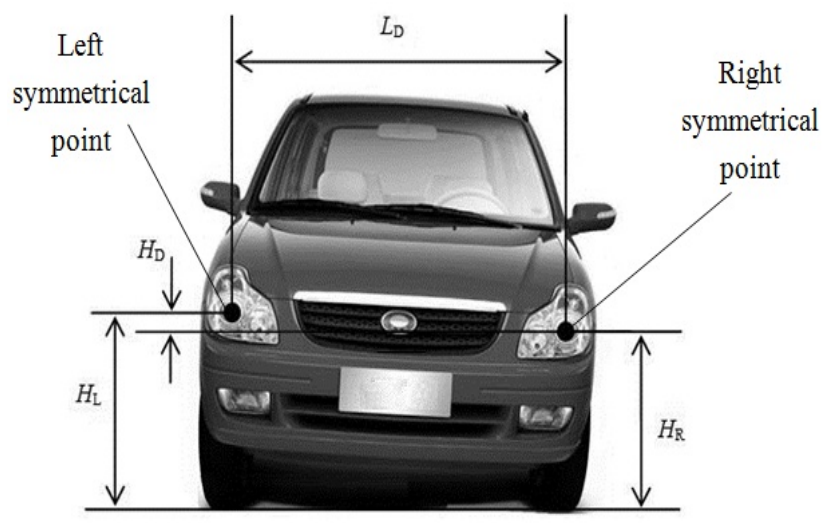

Fig (1). The detection principle of automobile body inclination angle.

\subsection{Detection Principle of Automobile Body Inclination Angle}

According to the requirements of automobile body inclination angle detection, the key of system realization is to select appropriate symmetrical points in the car body, measure the distance difference between the two points and the ground.

There are a variety of ways to select the symmetrical points, such as the intersecting points of mirrors and car body, the most prominent parts of the auto body, and so on. For the convenience of fusing this measurement together with automobile headlamp detection and other items, the feature points of the headlamp areas are chosen as the symmetrical points of the automobile body.

The detection principle of car body inclination angle is shown in Fig. (1), $H_{\mathrm{L}}$ and $H_{\mathrm{R}}$ are respectively the heights of the left and the right symmetrical point above the ground, $H_{\mathrm{D}}$ is the height difference between two symmetrical points, as shown in Eq. 1.

$H_{\mathrm{D}}=H_{\mathrm{L}}-H_{\mathrm{R}}$

Supposing that the horizontal distance between the two symmetrical points is $L_{\mathrm{D}}$, then, the inclination angle of the automobile body can be gotten, as shown in Eq. 2 .

$\theta=\arctan \left(\frac{H_{D}}{L_{D}}\right)$

If $\theta$ is positive, it indicates that the car inclines to right, and vice versa.

\subsection{Design of Automobile Body Inclination Angle Detec- tion Scheme}

According to the detection principle of automobile body inclination angle, the measurement is realized based on digital image processing technology [16]. The detection system is mainly composed of the tested automobile, single camera and computer. The car is parked in the detection position and the camera is set in front of it. After camera calibration, the image plane is vertical to the detection field and parallel to the front face of the vehicle. The captured image is transmitted to the computer which processes the image, extracts symmetrical points, calculates the horizontal distance and height difference, and ultimately analyzes the car body inclination angle.

The concrete process of system realization is as follows:

\section{(1) Camera calibration}

In this study, the camera is calibrated based on the method of Zhang $[17,18]$ which uses planar target and the calibration process is convenient. The camera position is adjusted to let the camera align with the car and the testing field strictly.

\section{(2) Image acquisition}

The automobile body inclination angle detection system is constructed and the image of automobile front-face is captured through a single camera.

\section{(3) Image preprocessing}

The automobile image is transferred to the computer through USB (Universal Serial Bus) interface, and it is preprocessed by the computer software to obtain the gray scale image.

(4) Automobile body symmetrical points detection

This step is the key process to measure the inclination angle. Firstly, the likely location of the car license plate is estimated based on the camera calibration results and the distance between the camera and the car, the modified Hough transform based on the fixed ratio of height to width is proposed to detect rectangle and locate the front license plate position accurately. Then, according to the location of the license plate, the general headlamp locations are estimated and the headlamp detection areas are constructed, the special lines in these areas are detected by using the modified Hough transform based on distance constraint and angle constraint. Lastly, the feature points of the automobile headlamps are extracted.

\section{(5) Automobile body inclination angle analyzing}

According to the pair of the detected left and right headlamp feature points, the height difference and horizontal distance are calculated and the automobile body inclination angle is analyzed.

\section{FRONT LICENSE PLATE LOCATION}

Because of the complexity of automobile structure, different models of cars have different shapes, different sizes, and different colours and textures. If the whole image of the automobile is analyzed to query symmetrical points, it needs a huge amount of calculation, the realization is also very difficult and inefficient, and then the precision, accuracy and real-time of the detection are affected.

In order to quickly and accurately extract the body symmetrical points, this study makes the license plate as detection reference. Firstly, the system locates the license plate, and then finds out the likely body symmetrical points. The license plate location can be divided into two steps: coarse positioning and fine positioning. The former means that the approximate position is previewed according to certain information, such as image pixels, camera focal length, and so 
on. The latter is only to analyze the rough location area to extract the exact position of the license plate.

To extract and locate the vehicle's license plate, it is necessary to understand its characteristics, especially the distinctive features comparing with the automobile body [19]. As the identity card of an automobile, the manufacture and use of the license plate are strictly specified [20]. According to the public safety industry standards of China, the standard size of the vast majority of front license plates, including small cars, is $440 \mathrm{~mm} \times 140 \mathrm{~mm}$, and its foreground and background colours are ruled specifically [21]. It can be seen that the license plate has fixed length to width ratio which value is $r=3.143$. Because of the distinctive feature, the license plate location has been widely used in the automotive testing field, such as intelligent transportation, vehicle tracking, etc. [22].

There are several ways to locate the front license plate, such as, making use of the texture and colour of the license plate, projection method, and so on [23]. In this study, it is required that the camera is aligned with the front face of the automobile, then, the rectangle of the license plate with certain ratio is vertical to the ground, that is, the long sides are horizontal and the short sides are vertical. With these features, a special rectangular is detected based on modified Hough transform to locate the license plate precisely.

\subsection{Basic Idea of Hough Transform}

Hough [24] proposed a method of finding and chaining edge pixels by using image global characteristic, which is called Hough transform. It is a line description method that a line expressed by rectangular coordinates in an image space can be transformed into a point in polar coordinates. The detection result based on Hough transform is less affected by the noise and curve interruption. The transform is widely used in line positioning and direction detection, and it can be extended to the detection of given shapes including rectangle, ellipse, and so on.

In the rectangular coordinates of the image space, a line through the point $(x, y)$ can be expressed, as shown in Eq. 3.

$$
y=a x+b
$$

Where, $a$ is the slope and $b$ is the intercept.

Since the value of slope $a$ can be infinite in a straight line space, which is difficult to express. Then, the line can be expressed by polar coordinates, as shown in Eq. 4 .

$\rho=x \cos \theta+y \sin \theta$

Where, $\rho$ is the normal distance from the origin to the line, $\theta$ is the angle between the axis $x$ and the normal. In this way, a point in image space corresponds to a curve in $(\theta, \rho)$ plane.

The points of the same line in image space correspond to the intersection point of the curves in the parameter space, and this feature can be used to detect collinear points. Similarly, all the lines intersected at a same point in the parameter space have corresponding collinear points in the image space [25].
Hough transform converts the task of line detection in image space to point detection in the parameter space. It can be completed through the relatively simple accumulation statistics operation. The basic steps of Hough transform are as follows.

(1) The original image is preprocessed to obtain a binary image, which is a set of edge feature points.

(2) The plane $(\theta, \rho)$ is subdivided and an accumulator array is generated, and the initial value of every unit is set to 0 .

(3) Each point of the image is calculated, and the corresponding accumulator units are updated.

(4) The local peak points of the accumulator are found.

(5) According to these peak points, the relative image pixels are searched and connected into line segments.

According to the line detection process based on Hough transform, it can be analyzed that the detection accuracy is mainly affected by the quantization intervals of parameter space and line connection ways. This method has some shortcomings that it has a large number of parameters to select, and must quantize the parameter space in advance. Then, this method needs large storage space and computation.

\subsection{Automobile License Plate Detection Based on Height to Width Ratio Constraint}

According to the characteristics of the Hough transform, when camera is aligned with automobile, the rectangle of the car front license is transformed that its horizontal and vertical side edges can respectively produce two peaks when $\theta$ is about $90^{\circ}$ or $0^{\circ}$ in the parameter space. The distance between the former two peak points is about $r$ times of that between the latter two points [26]. Thus, the ratio $r$ can be used to eliminate the interference of those peaks which are not caused by license plate frames in the $(\theta, \rho)$ space [27]. When performing the Hough transform, by limiting $\theta$ in the horizontal or vertical direction, the calculation amount can be reduced and the analysis efficiency can be improved [28].

The detection steps of automobile front license plate are as follows:

(1) The front license plate detection area is pre-located according to the camera calibration results.

(2) The image is transformed, that is, $f(x, y) \rightarrow H(\theta, \rho)$.

(3) In the transform space, several local peaks corresponded by horizontal edges at $\theta=90^{\circ}$ are found, and the distances between every two points are computed to construct a set $d n_{p q}$.

(4) Likewise, a number of local peak points corresponded by vertical edges at $\theta=0^{\circ}$ are detected, and the distances between every two points are calculated to form another set $d z_{s t}$. 


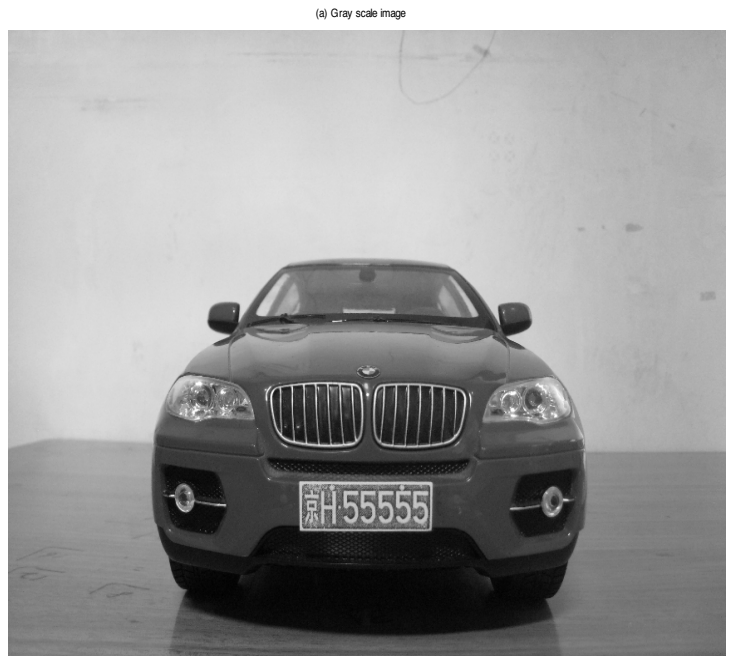

Fig (2). The gray scale image of a model car with simulation license plate.

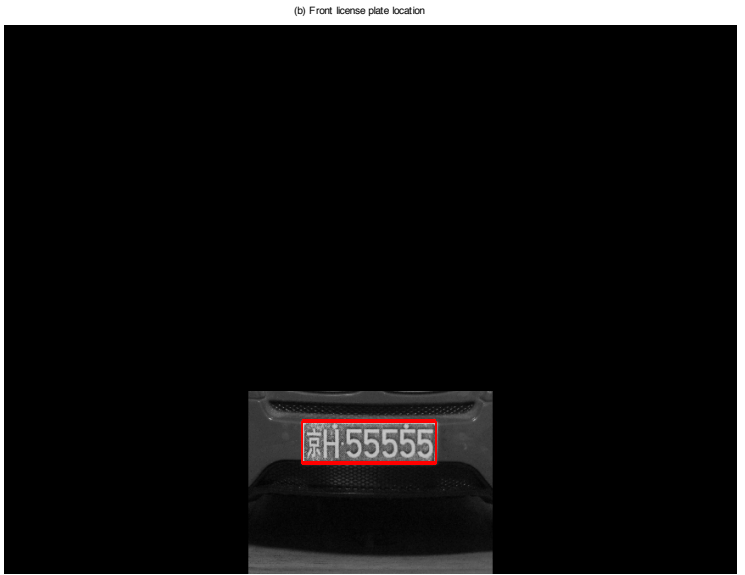

Fig. (3). The result of front license plate location of the model car.

(5) Taking one respective distance from the two distance sets to compare them, the pair which ratio is nearest to $r$ is selected, and then the two distances correspond to four points which are the four diagonal points of the front license plate.

(6) According to the selected four peaks in Hough transform domain, the corresponding lines can be gotten in the original image space, and a rectangle is found, that is the outline of the automobile license plate, and then the front license plate positioning is completed.

The gray scale image of a model car with simulation license plate is processed by the above process, and the front license plate is located, as shown in Fig. (2) and Fig. (3).

\section{AUTOMOBILE BODY SYMMETRICAL POINTS DETECTION}

\subsection{Selection of Body Symmetrical Points}

The car itself is symmetrical about the longitudinal axis, in theory, there are a variety of methods to select the symmetrical points, but the relevant studies are few. In order to improve the detection accuracy and efficiency, it is

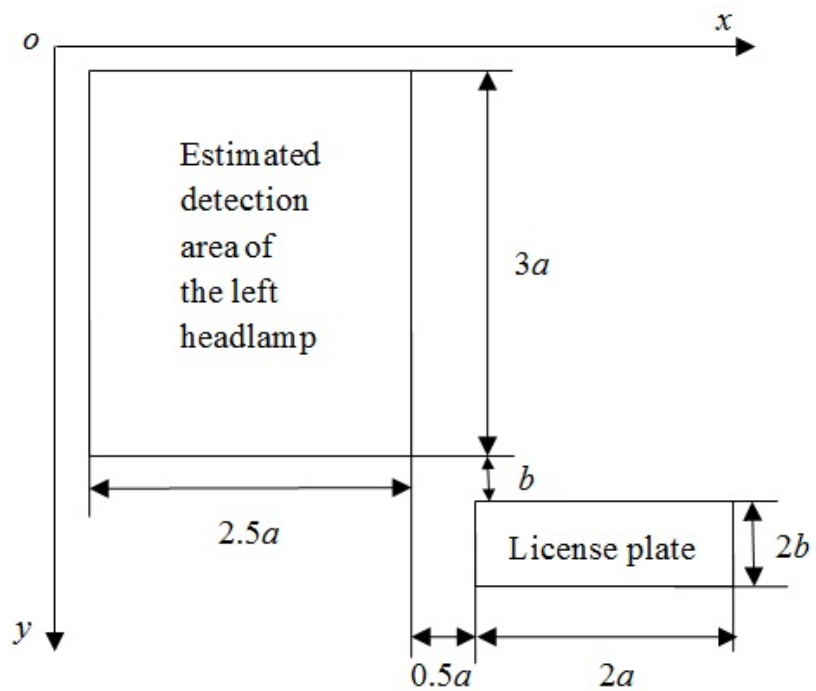

Fig. (4). The relative location relation between the front license plate and the left headlamp detection area.

necessary that the symmetrical points have distinct features. Moreover, the distance between two symmetrical points is moderate and not too small. By comprehensive consideration, this study selects headlamps as the extraction areas of the symmetrical points.

According to the location result of automobile license plate, the general detection areas of headlamp are estimated firstly, the feature points are extracted only in these regions to reduce the computation amount and to improve the detection real-time. Based on the statistics and analysis of the relative position relationship of the headlamps and the front license plate of many vehicles, the headlamp detection areas are predicted. The relative approximate location relationship between the front license plate and the left headlamp detection area is shown in Fig. (4). The detection area maybe not able to cover the whole left headlamp, but can contain the feature points of interest. Similarly, the right headlamp detection area can be gotten according to the position relation between the front license plate and the right headlamp.

After pre-estimation of headlamp detection areas, it is need to extract the symmetrical points of the automobile body in these areas. The extracted feature points can be corners, or the centers of the headlamps. According to their shapes, the headlamps can be divided into three types: circular, rectangular and alien headlamp, their typical shapes are shown in Fig. (5). Most automobiles adopt semi-enclosed alien headlamps, most of which are flat and long in shape. These headlamps are usually embedded into body integrally to form the automobile surface, which is conducive to forming streamlined body, and it is beautiful and practical.

For circular and rectangular headlamps, because of their regular shapes, the image segmentation method can be used to obtain the headlamp areas and then to analyze their centers. But these two types of headlamps are rarely used in automobiles, and then this detection method is not representative. Since this study mainly detects the small cars, by analyzing the headlamp shapes, most alien headlamps have a certain angle on their inside, so the inside corners can be selected as the feature points. What is more, this method is 


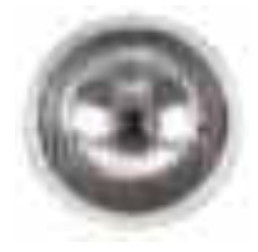

(a) Circular

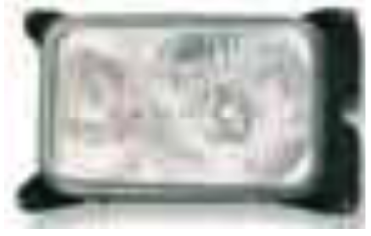

(b) Rectangular

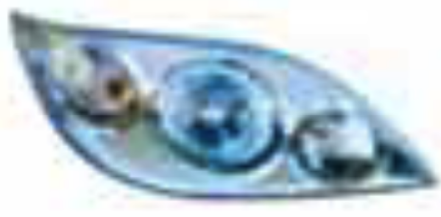

(c) Alien

Fig. (5). The typical shapes of three types of headlamps.

also applicable to the rectangular headlamps. Taken together, in order to hold the consistency of the detection methods, for alien and rectangular headlamps, the inside corners are made as the feature points; for circular headlamps, the circles or oval contours are extracted and analyzed to make the centers of mass as the feature points.

To achieve the automation of the detection process, the detected feature points of headlamps are divided into two categories: circle and corner. Since the characteristic of the circle is more outstanding, firstly, the headlamp areas are analyzed to check if there are circles with certain characteristic, if there are, their centers are extracted, if not, the corners are detected. Whether corners or circular contours, they can be detected based on Hough transform [29]. This study mainly analyzes the method of detecting corners for rectangular or alien headlamps.

\subsection{Angle and Distance Constraints for Line Detection}

By analyzing the rectangular and alien headlamps as shown in Fig. (5), it can be seen that there are at least two corner points in the left headlamp detection area: the top left corner and lower right corner. This study only detects the latter which location is labeled by point $\mathrm{P}$, as shown in Fig. (6). In order to detect the corner, the lines 1 and 2 should be extracted, and the coordinate of the corner can be acquired according to the equations of the two specified lines.

According to the outline characteristics of the headlamp areas, Hough transform is used to extract the two lines. In order to extract the feature points rapidly, the angle and

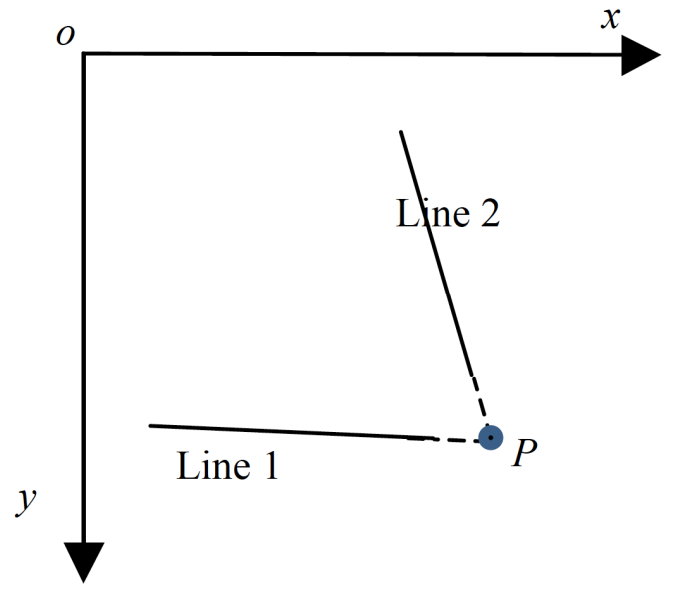

Fig. (6). The detection diagram of the lower right corner of the left headlamp detection area. distance constraints for the two lines should be created. The left headlamp is made as an example to explain the corner detection process.

\subsubsection{Angular Constraint}

The line 1, as shown in Fig. (6), is approximately horizontal, the angle constraint can be established for the angle between the axis $x$ and the normal of line 1. It has two ranges: $\left[-90^{\circ},-85^{\circ}\right]$ and $\left[85^{\circ}, 90^{\circ}\right]$.

There are two cases for line 2, for the rectangular headlamps, it is basically perpendicular to the $x$-axis which angle is close to 0 and the constraint is $\left[-5^{\circ}, 5^{\circ}\right]$; for the alien headlamps, the angle vary greatly and the constraint can be [$\left.45^{\circ},-5^{\circ}\right]$. Combining these two cases, the angle constraint on line 2 is $\left[-45^{\circ}, 5^{\circ}\right]$.

According to the angle constraints, the candidate sets for the two lines can be extracted respectively.

\subsubsection{Distance Constraint}

For different types of automobiles, the distance between the origin point and the left headlamp changes a lot, and then the distance constraints between the origin and the two lines are difficult to create. But, since the ultimate goal of this study is to extract the corners, and the distance between line 1 and line 2 is least, in other words, the right end of line 1 is nearest to the bottom end of line 2, ideally the two lines even intersect at the corner point. According to this relationship, the distance constraint on the two lines can be created.

Similarly, for the right headlamp detection area, the lower left corner is detected as feature point and the corresponding angle and distance constraints can be obtained.

\subsection{Line Detection Based on Truncated Hough Trans- form}

In the line detection process based on Hough transform, the detected points do not fall entirely on the straight line, but they are distributed in the line-centered neighborhoods [30]. In this study, for a corner of the rectangular or alien headlamp, it is not usually formed by the intersection of those two straight lines, but by cures with certain curvature. Therefore, in the process of quantization, if the interval is slightly larger, great error can be brought. On the contrary, if the interval is too small, the accuracy can be improved, but the computation amount increases rapidly. It is necessary to find a balance between the two cases. 


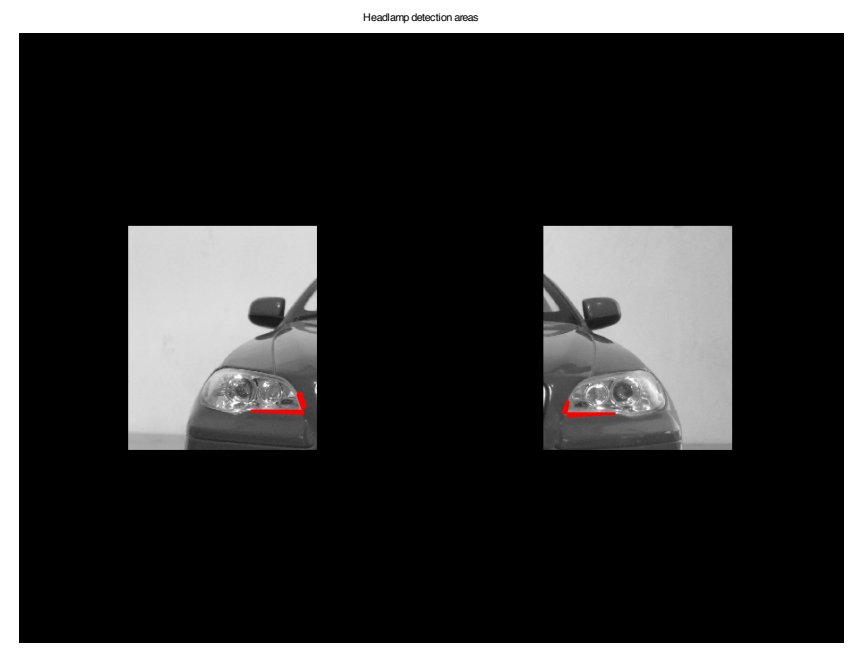

Fig. (7). The extracted four line segments of the headlamp detection areas of the model car.

In this study, under the premise of guaranteeing the detection real-time, on the basis of extracting the local maximal peaks and finding the corresponding pixels, combining the characteristics of lines in the headlamp areas, a line extraction method based on truncating the line pixels is proposed. The implementation process is as follows:

(1) According to an extreme point, the corresponding pixels are searched.

(2) According to the shape's characteristics of headlamp corner, the detected pixels are divided into three parts: $C_{1}$ is the set of pixels locating on the middle portion of the line, occupying about $60 \% ; C_{2}$ and $C_{3}$ are sets of pixels on each side of $C_{1}$, contributing about $20 \%$ respectively.

(3) The pixels in $C_{1}$ are connected into a line and the precise slope is calculated, which is denoted by $k$.

(4) Starting from the nearest pixel of $C_{2}$ to $C_{1}$, the slope of each point is calculated, if the difference between the slope and $k$ is less than a specified threshold value, the pixel is preserved and the next pixel will be analyzed again; otherwise, the pixel and pixels after it are discarded.

(5) The pixels in $C_{3}$ are operated like step (4).

(6) The pixels of the three updated sets are connected to form the final extracted line segment.

Since the number of pixel to be processed decreases, this method can improve the detection accuracy and the computational complexity does not change significantly.

Based on the above distance and angle constraints and pixel truncating operation, the extracted four line segments of the two headlamps of the model car can be obtained, as shown in Fig. (7).

\subsection{Analysis of Automobile Body Inclination Angle}

Based on the above analysis, the detected lines from the left and right headlamp areas are analyzed respectively, and the line equations are constructed to identify two intersection points, that is, the two symmetrical points of automobile body are gotten, their coordinates are respectively $(895,1209)$ and $(1710,1220)$.

According to Eq. 2, the inclination angle of the model car body is as follows.

$\theta=\arctan \left(\frac{1220-1209}{1710-895}\right)=0.773^{\circ}$

Since $\theta>0$, the model car inclines to right. According to the detection requirement, the body inclination angle is within the allowable scope.

In order to verify the detection method, this study does several experiments to measure the inclination angle of the same model car. The experimental results are shown in Table 2. The average of the measued inclination angles is $0.810^{\circ}$. The absolute value of the maximum error is $0.198^{\circ}$ and less than $0.2^{\circ}$ which can meet the requirement of the measurement system.

\section{CONCLUSION}

On the basis of analyzing several methods of measuring the symmetrical points height difference and inclination angle of automobile body and summarizing their advantages and disadvantages, the modified Hough transform is proposed to detect lines and rectangles, the method of computing the automobile body inclination angle based on headlamp area detection is brought forward and the inclination angle detection system is constructed and realized.

The experiment results show that the Hough transform based on the constraint on length to width ratio can detect the certain rectangle in the estimated front license plate detection area and the vehicle license plate can be located quickly and exactly; in the estimated headlamp detection areas, the lines can be extracted effectively by Hough transform based on the constraints on angle and distance, then the two feature points of the headlamps are gotten, and the operation amount can be greatly deceased; according to the detected feature points, the height difference of the symmetrical points and the inclination angle of automobile body can be obtained.

The scheme is achieved based on digital image processing technology, as a non-contact measurement method, it has better real-time performance. At the same time, the measurement can link seamlessly with the current used vehicle testing stations, and can be integrated with other testing items and can realize fully automatic detection. The

Table 2. The measurement results of seven experiments (unit: ${ }^{\circ}$ ).

\begin{tabular}{|c|c|c|c|c|c|c|c|}
\hline Experiment No. & $\mathbf{1}$ & $\mathbf{2}$ & $\mathbf{3}$ & $\mathbf{4}$ & $\mathbf{5}$ & $\mathbf{6}$ & $\mathbf{7}$ \\
\hline \hline Inclination angle & 0.773 & 0.626 & 1.018 & 1.013 & 0.657 & 0.894 & 0.689 \\
\hline
\end{tabular}


measurement data can provide foundation for automobile performance testing and maintenance, and this study have certain theoretical and practical values.

Through the process of detecting the height difference of symmetrical points and analyzing the inclination angle of automobile body, the measurement precision and accuracy depend on the detection algorithm of symmetrical feature points and the detecting method of lines and rectangles. Therefore, in order to improve the detection accuracy and practicality, more precise line detection algorithms are the next research objectives. In addition, this study only chooses headlamps as feature points of the automobile body, then, the method of selecting new symmetrical points is another future study direction.

\section{CONFLICT OF INTEREST}

The author confirms that this article content has no conflict of interest.

\section{ACKNOWLEDGEMENTS}

This study is supported by the Science and Technology Development Planning Project of Shandong Province (No. 2013 YD05004), People's Republic of China.

\section{REFERENCES}

[1] J. L. Liu, H. Wu, and Q. G. Wang, "Problem Analysis and settlement scheme of bodywork incline of bus and coach," Bus and Coach Technology and Research, no. 2, pp. 40-41, 2007.

[2] S. Y. Wang, "Study on the Form a Deviation of the Automobile Bodywork Examination Instrument," M.S. thesis, Jilin University, Changchun, China, 2005.

[3] Z. Y. Qian, "Research on Non-Contact Height Difference Measurement Machine," M.S. thesis, Tianjin University, Tianjin, China, 2005.

[4] C. T. Cheng, K. W. Chau, Y. G. Sun, and J. Y. Lin, "Long-term prediction of discharges in Manwan Reservoir using artificial neural network models," Lecture Notes in Computer Science, vol. 3498 , pp. 1040-1045, 2005.

[5] K. W. Chau, "Application of a PSO-based neural network in analysis of outcomes of construction claims," Automation in Construction, vol. 16, 5, pp. 642-646, 2007.

[6] C. L. Wu, K. W. Chau, and Y. S. Li, "Predicting monthly stream flow using data-driven models coupled with data-preprocessing techniques," Water Resources Research, vol. 25, no. 8, Res, 45, W08432 2009

[7] J. Zhang, and K. W. Chau, "Multilayer ensemble pruning via novel multi-sub-swarm particle swarm optimization," Journal of Universal Computer Science, vol. 15, no. 4, pp. 840-858, 2009.

[8] R. Taormina, K. W. Chau, and R. Sethi, "Artificial neural network simulation of hourly groundwater levels in a coastal aquifer system of the Venice lagoon," Engineering Applications of Artificial Intelligence, vol. 25, no. 8, pp. 1670-1676, 2012.

[9] W. J. Lou, "Study on Detecting Automobile Body Height Difference between Left and Right," M.S. thesis, Jilin University, Changchun, China, 2006.

[10] W. J. Lou, and L. G. Ma, "Research on the tester of automobile body height difference between left and right," China Science and Technology Information, no. 24, pp. 65-67, 2006.
[11] X. Y. Zhao, "Study on the detecting instrument of an automobile altitude right-and-left difference," M.S. thesis, Jilin University, Changchun, China, 2010.

[12] H. Y. Zhang, and J. Su, "Design of non-contact tester for altitude difference of automobile body's right-and-left symmetrical point," Machinery Design \& Manufacture, no, 2, pp. 134-135, 2008.

[13] H. Y. Lin, "Study on Testing System of the Position Deviation of Vehicle-body and Axle Based on Stereo Vision," Ph. D. thesis, Jilin University, Changchun, China, 2008.

[14] GB18565-2001. "Multiple performance requirement and detecting methods for commercial vehicles," Standardization Administration of the People's Republic of China, China, 2011.

[15] GB1589-2004. "Limits of dimensions, axle load and masses for road vehicles," Standardization Administration of the People's Republic of China, China, 2004.

[16] Z. K. Huang, and K. W. Chau, "A new image thresholding method based on Gaussian mixture model," Applied Mathematics and Computation, vol. 205, no. 2, pp. 899-907, 2008.

[17] Z. Zhang, "Flexible camera calibration by viewing a plane from unknown orientations," The Proceedings of the $7^{\text {th }}$ IEEE International Conference on Computer Vision, September 20-27, Kerkyra, Greece, pp. 666-673, 1999.

[18] Z. Zhang, "A flexible new technique for camera calibration," IEEE Transactions on Pattern Analysis and Machine Intelligence, vol. 22, no. 11, pp. 1330-1334, 2000.

[19] C. Yang, and D. C. Yang, "License plate location algorithm in the intelligent transportation system," Electronic Measurement Technology, vol. 30, no. 12, pp. 52-56, 2007.

[20] Y. P. Yang, "Study on Automobile License Plate Recognition System Based on Image Processing," M.S. thesis, Shenyang Ligong University, Shenyang, China, 2009.

[21] GA36-2007. "License plate of motor vehicle of the People's Republic of China," The Ministry of State Security of the People's Republic of China, China, 2007.

[22] S. Q. Mao, X. H. Huang, and M. Wang, "An adaptive method for Chinese license plate location," Proceedings of the $8^{\text {th }}$ World Congress on Intelligent Control and Automation, July 7-9, IEEE, Jinan, China, 2010, pp. 6173-6177.

[23] Y. X. Su, and J. J. Luo, "License location technology based on edge detection and image subtraction methods," Instrumentation Technology, no. 4, pp. 40-41, 44, 2008.

[24] P. V. Hough, "Method and means for recognizing complex patterns," U.S. Patent, 3069654, March 25, 1962.

[25] G. K. Chen, "Line detection based on hough transform," Journal of Xi'an Aero technical College, no. 3, pp. 34-36, 2007.

[26] D. Liang, J. Gao, Q. Z. Fu, and Y. C. Chen, "License plate area location and reconstruction based on shape feature and inverse hough transform," Computer Applications, vol. 22, no. 5, pp. 4344, 47, 2002.

[27] W. C. Jiang, and S. L. Zheng, "Research on vehicle license plate recognition system based on hough transform and neural network," Journal of Anqing Teachers College (Natural Science Edition), vol. 16 , no. 3, pp. 52-55, 2010.

[28] V. Kamat, and S. Ganesan, "An efficient implementation of the Hough transform for detecting vehicle license plates using DSP'S," Proceedings of Real-Time Technology and Applications Symposium, May 15-17, Chicago, IL, IEEE, 1995, pp. 58-59, 1995.

[29] K. H. Qin, H. Y. Wang, and J. T. Zheng, "A unified approach based on hough transform for quick detection of circles and rectangles," Journal of Image and Graphics, vol. 15, no. 1, pp. 109-115, 2010.

[30] H. J. Wang, L. Q. Chen, and L. Wang, "Extraction and correction of rectangle geometric date in machine vision," Journal of Mechanical and Electrical Engineering, vol. 27, no. 4, pp. 95-96,111, 2010. 